\title{
Evaluation of Arteriosclerosis Using the Brachial-Ankle Pulse Wave Velocity in Patients with Visceral Lesion-Free Systemic Lupus Erythematosus Characterized by Skin Lesions
}

\author{
Kimiko Maruyama, Takaharu Ikeda, Katsunori Tanaka and Fukumi Furukawa*
}

Department of Dermatology, Faculty of Medicine, Wakayama Medical University, Japan

\begin{abstract}
Recently, the cardio-/cerebrovascular lesion-related mortality rate has been high in patients with systemic lupus erythematosus (SLE). In these patients, the risk of cardio-/cerebrovascular lesions is also higher than in the general population. Cardio-/cerebrovascular lesions may occur during long-term follow-up. In this study, we evaluated arteriosclerosis using the brachial-ankle pulse wave velocity (baPWV) in visceral lesion-free SLE patients with skin lesions. In these patients, baPWV was higher than in healthy adults even at a young age. This suggests that baPWV is a possible tool to evaluate the patient's vascular function, which was difficult to evaluate using a conventional sera arteriosclerosis index. Even when conditions are characterized by skin lesions, it may be important to consider the influence on the cardio-/cerebrovascular systems, as indicated for patients with systemic symptoms.
\end{abstract}

Keywords: Arteriosclerosis, brachial-ankle pulse wave velocity, cutaneous lupus erythematosus, systemic lupus erythematosus.

\section{INTRODUCTION}

Previously, the frequent causes of death in patients with systemic lupus erythematosus (SLE) included nephropathy, central nervous system (CNS) lupus, and infectious diseases [1-3]. However, advances in clinical examination methods have facilitated the early diagnosis of SLE, and early treatments with corticosteroids or immunosuppressive drugs were established. Based on the background, there have recently been marked changes in the causes of death. According to the report of Bernatsky et al., angiopathy accounts for approximately $25 \%$ of the mortality [4]. It results from arteriosclerosis in many cases. Manzi et al. reported that atherosclerosis progressed from a young age in patients with SLE, and that atherosclerotic plaques were detected in $40 \%$ of patients. They indicated that the relative risk of atherosclerosis was 5 times higher in SLE patients, and that it was 50 times higher in those aged under 45 years [5]. Therefore, the assessment and treatment of arteriosclerosis are particularly important in SLE patients involving a high proportion of young patients.

To evaluate arteriosclerosis, a blood test is basically performed. As indices of arteriosclerosis, the (total cholesterol (T-Cho)-HDL)/HDL and LDL/HDL ratios are used. The blood test is simple. However, a study indicated that there was no correlation between the incidence of myocardial infarction in SLE patients and risk factors previously reported; evaluation based on the results of a blood test alone is incomplete [6]. In addition, there are some

*Address correspondence to this author at the Department of Dermatology, Wakayama Medical University, Postal Code: 641-0012, 811-1, Kimiidera, Wakayama City, Wakayama Prefecture, Japan; Tel: 81-73-441-0661;

Fax: 81-73-448-1908; E-mail: dajs@wakayama-med.ac.jp patients primarily complaining of skin lesions among those with SLE, as a systemic disease. It should be examined whether or not arteriosclerosis is present in these patients.

Other evaluation methods include imaging procedures, represented by carotid ultrasonography [6] and electronbeam computed tomography (CT) [7], and measurement of the pulse wave velocity (PWV) [8]). Imaging procedures depend on examiners' skills and require a specific time. The results are influenced by the presence or absence of instruments. We used the brachial-ankle pulse wave velocity (baPWV) as a noninvasive examination procedure for a large number of patients in order to make an early diagnosis in clinical practice.

\section{SUBJECTS AND METHODS}

The subjects were 138 patients who had been treated in our department and from whom informed consent was verbally obtained. As a routine screening test, baPWV was measured once a year. They consisted of 29 patients with SLE meeting the new Systemic Lupus International Collaborating Clinics (SLICC) classification criteria 2012 (mean age: $47.8 \pm 12.0$ years, 3 males, 26 females), 27 with systemic sclerosis (SSc) (mean age: 67.64 \pm 12.8 years, 3 males, 24 females), and 85 with other dermal diseases (eczema and dermatitis) (19 males, 66 females). Initially, a cuff for the limbs, an electrocardiographic clip for the wrist, and a heart sound sensor were attached to subjects. Subject information (age, sex, and body weight) necessary for measurement were input. After 5 minutes rest, baPWV is determined by simultaneously measuring the blood pressure and pulse wave in the limbs. In the subjects, excluding one case, facial erythema, discoid-shaped erythema, and photosensitivity were primarily observed at the time of 
diagnosis. Although articular symptoms were noted in 50\% of the subjects, no patient showed any cardiovascular/central nervous symptoms or kidney dysfunction, which was same in 85 with other dermal diseases (eczema and dermatitis). Follow-up periods ranged from 1 to 7 years.

The baPWV was measured using a blood pressure-/pulse wave-testing device (OMRON COLIN Co., Ltd, Japan). This parameter increases with age. The values obtained by the single regression analysis were compared with the mean baPWV in each age group, and dissociation was evaluated based on the standard deviation; individual baPWV values, which differed among age/sex groups, were corrected with the rate of change, facilitating assessment from the same perspective [9].

To measure conventional indices of arteriosclerosis, the (T-Cho - HDL)/HDL and LDL/HDL ratios, a blood test was simultaneously conducted at the baPWV measurement point in 13 subjects. Verbal informed consent was obtained. These parameters were calculated. Statistical analysis was performed by unpaired t-test.

Concerning the correlation with corticosteroid therapy, the steroid dose over a 5-year period was reviewed. The initial dose during this period was added to the last dose, multiplied by 5 (years), and divided by 2 . The value was regarded as a coefficient (example: initial maintenance dose,
$10 \mathrm{mg}$; after 5 years, $5 \mathrm{mg}$; and after 10 years, $3 \mathrm{mg}$ : $(10+5)$ $5 / 2+(5+3) 5 / 2=57.5)$. When the initial dosing involved steroid pulse therapy, initial maintenance therapy was selected as the initial value.

After preliminary presentation of this study to our Ethics Committee, it was decided that ethical approval was not required because the method employed was standard. This study was performed in compliance with the ethical principles of the Declaration of Helsinki based on 2000 version.

\section{RESULTS}

The baPWV values in 29 patients with SLE characterized by skin lesions and those predicted by regression analysis are presented in Fig. (1). A comparison of the estimated baPWV values in SSc patients, which were calculated by regression analysis, with the mean in each age group is shown in Fig. (2). In the SLE patients, baPWV was high even at a young age. Among 29 patients, the standard deviation (SD) exceeded 2 in 13 patients and it exceeded one in 4 patients, respectively. In young SSc patients (30 to 39 years), there was no marked difference in comparison with the mean of healthy adults. However, the difference increased with age, and the standard deviation exceeded $2 \mathrm{SD}$ in more than $50 \%$ of the patients aged over 60 years.

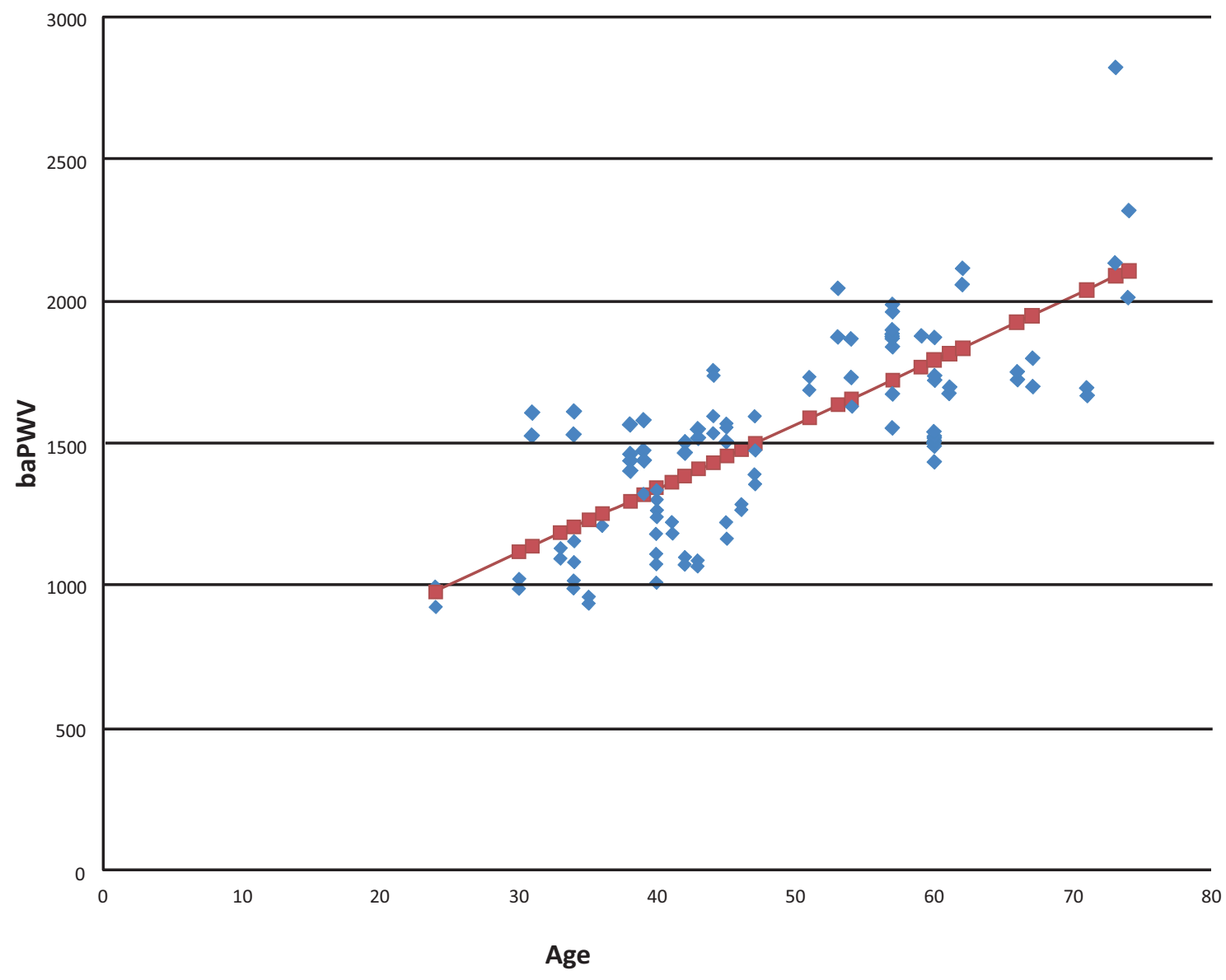

Fig. (1). Patient age and changes in baPWV. The predicted values, obtained by plotting the baPWV values in SLE patients and age on measurement and conducting regression analysis, are presented. 


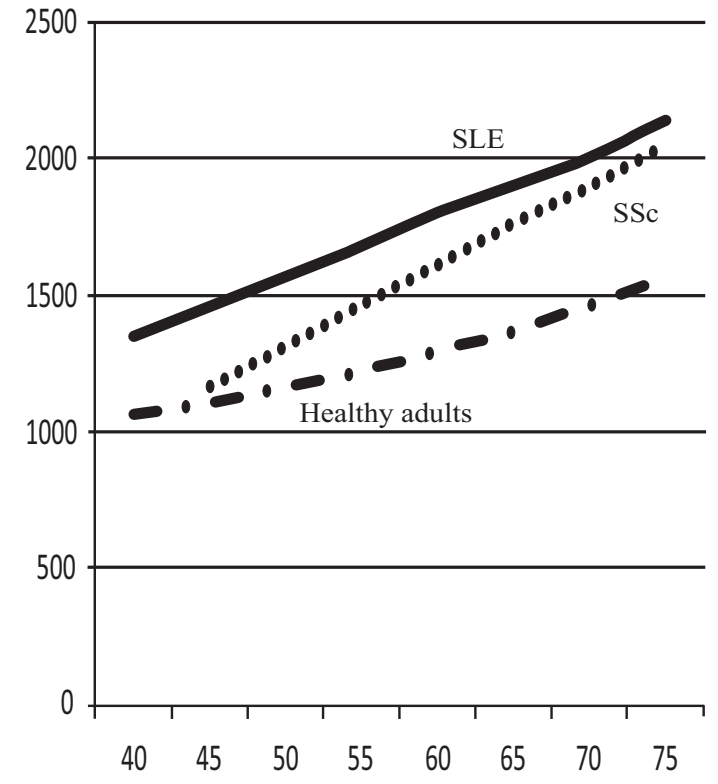

Fig. (2). The predicted values of baPWV in SLE, SSc patients and healthy adults with respect to age. The predicted values obtained through regression analysis in SLE and SSc patients were compared with the mean of 12517 healthy adults. The predicted values of baPWV in SLE and SSc (over 40 years old) and the mean of baPWV in the control (over 40 years old).

Comparison of baPWV in 13 patients with SLE, from whom informed consent regarding a blood test was obtained, with a conventional index of arteriosclerosis, the LDL/HDL ratio, is shown in Table 1. Comparison of (T-cho - HDL)/HDL with baPWV in the SLE patients is presented in Table 2.

Table 1. Standard deviation of baPWV and LDL/HDL in SLE patients.

\begin{tabular}{|c|c|c|c|}
\hline SDL/HDL & $<1 \mathrm{SD}$ & $\geq 1 \mathrm{SD},<2 \mathrm{SD}$ & $\geq 2$ SD \\
\hline$\leq 2$ & 3 & 1 & 2 \\
\hline$>2$ & 0 & 4 & 3 \\
\hline
\end{tabular}

The standard deviation of baPWV in SLE patients was classified by 1 and 2SD, and the LDL/HDL value was classified by 2 (a normal value).

Table 2. Standard deviation of baPWV and (T-cho - HDL)/ HDL in SLE patients.

\begin{tabular}{|r|c|c|c|}
\hline SD of baPWV & $<$ 1SD & $\mathbf{2 1 S D , ~ < 2 S D ~}$ & $\mathbf{2 2 S D}$ \\
\hline \hline$\leq 4$ & 3 & 4 & 3 \\
\hline$>4$ & 0 & 1 & 2 \\
\hline
\end{tabular}

A conventional index of arteriosclerosis, (T-cho - HDL)/HDL, were classified by 4 (a normal value).

Among 5 patients with baPWV exceeding $2 \mathrm{SD}$, the LDL/HDL ratio was normal in 2 and high in 3 (normal range $\leq 2$ ). Among 5 patients with baPWV from1SD to 2SD ( $\geq 1 \mathrm{SD}$, $<2 \mathrm{SD})$, the LDL/HDL ratio was normal in 1 and high in 4. Among 3 patients with normal baPWV $(<1 \mathrm{SD})$, the
LDL/HDL ratio was normal in 3 . Among the patients with baPWV more than $1 \mathrm{SD}$, the LDL/HDL ratio was relatively high but there were no statistical differences (by unpaired $t$ test).

Among 5 patients with baPWV exceeding 2SD, the (Tcho - HDL)/HDL ratio was normal in 3 and high in 2 (normal range $\leqq 4$ ). Among 5 patients with baPWV from $1 \mathrm{SD}$ to $2 \mathrm{SD}(\geq 1 \mathrm{SD},<2 \mathrm{SD})$, the (T-cho - HDL)/HDL ratio was normal in 4 and high in 1 . Among 3 patients with normal baPWV, (T-cho - HDL)/HDL was normal in 3. When compared with the association of baPWV and LDL/HDL ratio, the inverse correlations were observed among groups of $\geq 1 \mathrm{SD},<2 \mathrm{SD}$ and $\geq 2 \mathrm{SD}$ ( $\mathrm{p}<0.01$, unpaired t test)

As shown in Table $\mathbf{3}$, there was no correlation between the coefficient of corticosteroid therapy and baPWV.

Table 3. Dose of corticosteroid (coefficient) and baPWV (as SD) in patients with SLE.

\begin{tabular}{|c|c|c|c|}
\hline Coefficient & $<\mathbf{1 S D}$ & $\geq \mathbf{1 S D}, \mathbf{2} \mathbf{S D}$ & $\mathbf{2}$ SD \\
\hline \hline $0-50$ & 4 & 0 & 5 \\
\hline $51-100$ & 2 & 2 & 2 \\
\hline $101-150$ & 2 & 3 & 2 \\
\hline $151-200$ & 0 & 0 & 2 \\
\hline $201-250$ & 0 & 0 & 0 \\
\hline$>251$ & 0 & 1 & 2 \\
\hline Total & 8 & 6 & 13 \\
\hline
\end{tabular}

There was no statistically significant correlation.

\section{DISCUSSION}

The results of this study involving 29 patients with SLE characterized by skin lesions suggest that the influence on the cardio-/cerebrovascular systems must be considered even when there are no systemic symptoms.

In this study, baPWV exceeded the mean +1 to $+2 \mathrm{SD}$ of healthy adults in many subjects aged 40 years or older. In some patients with SLE, baPWV was high even when conventional risk factors for arteriosclerosis, the LDL/HDL ratio and (T-cho - HDL)/HDL, were normal. When baPWV was normal, the LDL/HDL ratio and (T-cho - HDL)/HDL were normal ( 2 and 4 , respectively). Interestingly these two ratios varied from baPWV value in our examined group (Tables 2 and 3). Reports by Roman [6], Asanuma et al. [7] and our study suggested that conventional management based on blood collection data alone is risky to determine the degrees of vascular damage.

Measurement of baPWV is a method to diagnose arteriosclerosis in which the principle that the pulse wave of blood ejected from the heart is accelerated with the stiffness of arteries was applied. Measurement is simple, and the duration of examination involving preparations is approximately 5 minutes. The baPWV facilitates the detection of early angiopathy. It reflects wall thickening of the arterial media. A study reported that baPWV was useful as an index of the grade of atherosclerosis or coronary risks, or as a parameter for predicting cardiovascular disease [10]. It is also possible to evaluate aortic sclerosis [11]. 
Thus, measurement of baPWV is a noninvasive method to manage/diagnose arteriosclerotic lesions in patients with SLE, and it may be useful in outpatient clinics. In addition, baPWV also tends to increase at an advanced age in patients with SSc. Therefore, this parameter should be considered for regular screening.

In SLE patients in whom baPWV was within the normal range at the first visit, we should continue the follow-up examination every year because as shown in Figs. (1, 2) SLE patients have high risk of the increasing in baPWV. Another reason is that baPWV reached the mean plus $1 \mathrm{SD}$ of healthy adults during at least 5 years follow up which is based on the preliminary analysis of the present studies.

This suggests that baPWV is a possible tool to evaluate the patient's vascular function, which was difficult to evaluate using a conventional sera arteriosclerosis index. Even when conditions are characterized by skin lesions, it may be important to consider the influence on the cardio/cerebrovascular systems, as indicated for patients with systemic symptoms.

\section{CONFLICT OF INTEREST}

The authors confirm that this article content has no conflict of interest.

\section{ACKNOWLEDGEMENTS}

\section{REFERENCES}

[1] Merrell M, Shulman LE. Determination of prognosis in chronic disease, illustrated by systemic lupus erythematosus. J Chronic Dis 1955; 1: 12-32.

[2] Dubois EL, Wierzchowiecki M, Cox MB, Weiner JM. Duration and death in systemic lupus erythematosus. An analysis of 249 cases. JAMA 1974; 227: 1399-402.

[3] Ofuji S, Miyawaki S. Epidemiological studies of systemic lupus erythematosus in Japan. Ryumachi 1975; 15: 310-25.

[4] Bernatsky S, Boivin JF, Joseph L, et al. Mortality in systemic lupus erythematosus. Arthritis Rheum 2006; 54: 2550-7.

[5] Manzi S1, Meilahn EN, Rairie JE, et al. Age-specific incidence rates of myocardial infarction and angina in women with systemic lupus erythematosus: comparison with the Framingham Study. Am J Epidemiol 1997; 145: 408-15.

[6] Roman MJ1, Shanker BA, Davis A, et al. Prevalence and correlates of accelerated atherosclerosis in systemic lupus erythematosus. N Engl J Med 2003; 349: 2399-406.

[7] Asanuma Y1, Oeser A, Shintani AK, et al. Premature coronaryartery atherosclerosis in systemic lupus erythematosus. N Engl J Med 2003; 349: 2407-15.

[8] Selzer F, Sutton-Tyrrell K, Fitzgerald S, Tracy R, Kuller L, Manzi $\mathrm{S}$. Vascular stiffness in women with systemic lupus erythematosus. Hypertension 2001; 37: 1075-82.

[9] Tomiyama H, Yamashina A, Arai T, et al. Influences of age and gender on results of noninvasive brachial-ankle pulse wave velocity measurement-a survey of 12517 subjects. Atherosclerosis 2003; 166: 303-9.

[10] Lehmann ED, Riley WA, Clarkson P, Gosling RG. Non-invasive assessment of cardiovascular disease in diabetes mellitus. Lancet 1997; 350(Suppl 1): 14-9.

[11] Lehmann ED. Clinical value of aortic pulse wave velocity measurement. Lancet 1999; 354: 528-9.

Declared none. 